\title{
Nonnegative solutions of a class of second order nonlinear differential equations
}

\author{
by S. STANĚK (Olomouc)
}

Abstract. A differential equation of the form

$$
\left(q(t) k(u) u^{\prime}\right)^{\prime}=\lambda f(t) h(u) u^{\prime}
$$

depending on the positive parameter $\lambda$ is considered and nonnegative solutions $u$ such that $u(0)=0, u(t)>0$ for $t>0$ are studied. Some theorems about the existence, uniqueness and boundedness of solutions are given.

1. Introduction. In [6] the equation

$$
\left(k(u) u^{\prime}\right)^{\prime}=f(t) u^{\prime}
$$

was considered and the author has given sufficient conditions for the existence and uniqueness of nonnegative solutions $u$ such that $u(0)=0, u(t)>0$ for $t>0$. This problem is connected with the description of the mathematical model of the infiltration of water. For more details see e.g. [3]-[5].

In [4] and [5] the existence and uniqueness of nonnegative solutions was proved for the differential equations

$$
\left(u u^{\prime}\right)^{\prime}=(1-t) u^{\prime} \quad(t \in[0,1])
$$

and

$$
\left(u u^{\prime}\right)^{\prime}=A^{-t} u^{\prime} \quad(A>1) .
$$

The methods are based on the special form of the equations and on the Banach fixed point theorem. In [1] and [2], the following equation was considered:

$$
\left(k(u) u^{\prime}\right)^{\prime}=(1-t) u^{\prime}
$$

1991 Mathematics Subject Classification: 34B15, 34C11, 34A10, 45G10.

Key words and phrases: nonlinear ordinary differential equation, nonnegative solution, existence and uniqueness of solutions, bounded solution, dependence of solutions on a parameter, boundary value problem. 
In this paper we consider the equation

$$
\left(q(t) k(u) u^{\prime}\right)^{\prime}=f(t) h(u) u^{\prime}
$$

which is a generalization of (1), and give sufficient conditions for the existence and uniqueness of solutions $u$ of (2) satisfying $u(0)=0, u(t)>0$ for $t>0$, as well as for their boundedness and unboundedness. In the last section we discuss the dependence of solutions of the equation $\left(q(t) k(u) u^{\prime}\right)^{\prime}=$ $\lambda f(t) h(u) u^{\prime}$ on the positive parameter $\lambda$ and we consider the boundary value problem $\left(q(t) k(u) u^{\prime}\right)^{\prime}=\lambda f(t) h(u) u^{\prime}, \lim _{t \rightarrow \infty} u(t ; \lambda)=a(\in(0, \infty))$. In accordance with [6] the proof of the existence theorem is based on an iterative method and a monotone behaviour of some operator. The proof of the uniqueness is different from the one in [6]. For the special case of (2), namely (1), we obtain the same results as in [6] (where $\int_{0}^{\infty}(k(s) / s) d s=\infty$ should be required).

2. Notations, lemmas. We will consider the differential equation (2) in which $q, k, f, h$ satisfy the following assumptions:

$\left(\mathrm{H}_{1}\right) \quad q \in C^{0}([0, \infty)), q(t)>0$ for all $t>0$ and $\int_{0} \frac{d t}{q(t)}<\infty$;

$\left(\mathrm{H}_{2}\right) \quad k \in C^{0}([0, \infty)), k(0)=0, k(u)>0$ for all $u>0$;

$\left(\mathrm{H}_{3}\right) \quad \int_{0} \frac{k(s)}{s} d s<\infty$ and $\int^{\infty} \frac{k(s)}{s} d s=\infty$;

$\left(\mathrm{H}_{4}\right) \quad f \in C^{1}([0, \infty)), f(t)>0, f^{\prime}(t) \leq 0$ for all $t \geq 0$;

$\left(\mathrm{H}_{5}\right) \quad h \in C^{0}([0, \infty)), h(u) \geq 0$ and the function $H(u):=\int_{0}^{u} h(s) d s$ is strictly increasing for all $u \geq 0$;

$\left(\mathrm{H}_{6}\right) \quad \int_{0} \frac{k(u)}{H(u)} d u<\infty$ and $\int^{\infty} \frac{k(u)}{H(u)} d u=\infty$.

By a solution of $(2)$ we mean a function $u \in C^{0}([0, \infty)) \cap C^{1}((0, \infty))$ such that $u(0)=0, u(t)>0$ for all $t>0, \lim _{t \rightarrow 0^{+}} q(t) k(u(t)) u^{\prime}(t)=0$, $q(t) k(u(t)) u^{\prime}(t)$ is continuously differentiable for all $t>0$ and (2) is satisfied on $(0, \infty)$.

For $u \in[0, \infty)$ we define the strictly increasing functions $K$ and $V$ by

$$
K(u)=\int_{0}^{u} k(s) d s, \quad V(u)=\int_{0}^{u} \frac{k(s)}{H(s)} d s .
$$


Clearly $K \in C^{1}([0, \infty)), V \in C^{0}([0, \infty)) \cap C^{1}((0, \infty)), \lim _{u \rightarrow \infty} K(u)=\infty=$ $\lim _{u \rightarrow \infty} V(u)$.

Set $M=\left\{u ; u \in C^{0}([0, \infty)), u(0)=0, u(t)>0\right.$ for $\left.t>0\right\}$.

LEMma 1. If $u$ is a solution of (2), then $u$ is a solution of the integral equation

$$
K(u(t))=\int_{0}^{t}\left(\frac{f(s)}{q(s)}-f^{\prime}(s) \int_{s}^{t} \frac{d z}{q(z)}\right) H(u(s)) d s
$$

and conversely, if $u \in M$ is a solution of (3), then $u$ is a solution of (2).

Proof. Let $u$ be a solution of (2). Integrating (2) from $a(>0)$ to $t$, we obtain

$$
\begin{aligned}
q(t) k(u(t)) u^{\prime}(t) & -q(a) k(u(a)) u^{\prime}(a)=\int_{a}^{t} f(s) h(u(s)) u^{\prime}(s) d s \\
& =f(t) H(u(t))-f(a) H(u(a))-\int_{a}^{t} f^{\prime}(s) H(u(s)) d s .
\end{aligned}
$$

Let $a \rightarrow 0^{+}$. We get

$$
(K(u(t)))^{\prime}=\frac{1}{q(t)}\left[f(t) H(u(t))-\int_{0}^{t} f^{\prime}(s) H(u(s)) d s\right]
$$

for $t>0$, and integrating (4) from 0 to $t$, we have

$$
\begin{aligned}
K(u(t)) & =\int_{0}^{t} \frac{1}{q(s)}\left[f(s) H(u(s))-\int_{0}^{s} f^{\prime}(z) H(u(z)) d z\right] d s \\
& =\int_{0}^{t}\left(\frac{f(s)}{q(s)}-f^{\prime}(s) \int_{s}^{t} \frac{d z}{q(z)}\right) H(u(s)) d s,
\end{aligned}
$$

and consequently, $u$ is a solution of (3).

Now, let $u \in M$ be a solution of (3). Then

$$
u(t)=K^{-1}\left[\int_{0}^{t}\left(\frac{f(s)}{q(s)}-f^{\prime}(s) \int_{s}^{t} \frac{d z}{q(z)}\right) H(u(s)) d s\right]
$$

for $t \geq 0$, where $K^{-1}$ denotes the inverse function to $K$ on $[0, \infty)$. From (4) it follows that $u^{\prime} \in C^{0}((0, \infty))$ and

$$
u^{\prime}(t)=\frac{1}{q(t) k(u(t))}\left[f(t) H(u(t))-\int_{0}^{t} f^{\prime}(s) H(u(s)) d s\right],
$$


therefore

(6)

$$
q(t) k(u(t)) u^{\prime}(t)=f(t) H(u(t))-\int_{0}^{t} f^{\prime}(s) H(u(s)) d s .
$$

Hence

$$
\begin{gathered}
\lim _{t \rightarrow 0^{+}} q(t) k(u(t)) u^{\prime}(t)=0, \quad q(t) k(u(t)) u^{\prime}(t) \in C^{1}((0, \infty)), \\
\left(q(t) k(u(t)) u^{\prime}(t)\right)^{\prime}=f(t) h(u(t)) u^{\prime}(t) \quad \text { for } t>0,
\end{gathered}
$$

consequently, $u$ is a solution of (2).

Remark 1. It follows from Lemma 1 that solving (2) is equivalent to solving the integral equation (3) in the set $M$.

Lemma 2. If $u \in M$ is a solution of (3), then

$$
V^{-1}\left(\int_{0}^{t} \frac{f(s)}{q(s)} d s\right) \leq u(t) \leq V^{-1}\left(f(0) \int_{0}^{t} \frac{d s}{q(s)}\right) \quad \text { for } t \geq 0 .
$$

Proof. Let $u \in M$ be a solution of (3). Then $u^{\prime}(t)>0$ for $t>0$ and (cf. (6))

$$
\begin{aligned}
f(t) H(u(t)) & \leq q(t) k(u(t)) u^{\prime}(t) \leq\left[f(t)-\int_{0}^{t} f^{\prime}(s) d s\right] H(u(t)) \\
& =f(0) H(u(t)),
\end{aligned}
$$

hence

$$
\frac{f(t)}{q(t)} \leq \frac{k(u(t)) u^{\prime}(t)}{H(u(t))}=(V(u(t)))^{\prime} \leq \frac{f(0)}{q(t)} \quad \text { for } t>0 .
$$

Integrating (8) from 0 to $t$, we obtain

$$
\int_{0}^{t} \frac{f(s)}{q(s)} d s \leq V(u(t)) \leq f(0) \int_{0}^{t} \frac{d s}{q(s)} \quad \text { for } t \geq 0
$$

and (7) follows.

Define the operator $T: M \rightarrow M$ by

$$
(T u)(t)=K^{-1}\left[\int_{0}^{t}\left(\frac{f(s)}{q(s)}-f^{\prime}(s) \int_{s}^{t} \frac{d z}{q(z)}\right) H(u(s)) d s\right] \quad \text { for } t \geq 0
$$

and set

$$
\underline{\varphi}(t)=V^{-1}\left(\int_{0}^{t} \frac{f(s)}{q(s)} d s\right), \quad \bar{\varphi}(t)=V^{-1}\left(f(0) \int_{0}^{t} \frac{d s}{q(s)}\right) \quad \text { for } t \geq 0 .
$$


Lemma 3. For $t \in[0, \infty)$,

$$
(T \underline{\varphi})(t) \geq \underline{\varphi}(t), \quad(T \bar{\varphi})(t) \leq \bar{\varphi}(t) .
$$

Proof. Setting

$$
\begin{aligned}
& \alpha(t)=\int_{0}^{t}\left(\frac{f(s)}{q(s)}-f^{\prime}(s) \int_{s}^{t} \frac{d z}{q(z)}\right) H(\underline{\varphi}(s)) d s-K(\underline{\varphi}(t)), \\
& \beta(t)=\int_{0}^{t}\left(\frac{f(s)}{q(s)}-f^{\prime}(s) \int_{s}^{t} \frac{d z}{q(z)}\right) H(\bar{\varphi}(s)) d s-K(\bar{\varphi}(t))
\end{aligned}
$$

for $t \geq 0$ we see that to prove Lemma 3 it is enough to show $\alpha(t) \geq 0$ and $\beta(t) \leq 0$ on $[0, \infty)$. Since

$$
\begin{aligned}
\alpha^{\prime}(t) & =\frac{f(t)}{q(t)} H(\underline{\varphi}(t))-\frac{1}{q(t)} \int_{0}^{t} f^{\prime}(s) H(\underline{\varphi}(s)) d s-K^{\prime}(\underline{\varphi}(t)) \underline{\varphi}^{\prime}(t) \\
& =-\frac{1}{q(t)} \int_{0}^{t} f^{\prime}(s) H(\underline{\varphi}(s)) d s \geq 0, \\
\beta^{\prime}(t) & =\frac{f(t)}{q(t)} H(\bar{\varphi}(t))-\frac{1}{q(t)} \int_{0}^{t} f^{\prime}(s) H(\bar{\varphi}(s)) d s-K^{\prime}(\bar{\varphi}(t)) \bar{\varphi}^{\prime}(t) \\
& \leq \frac{f(t)-f(0)}{q(t)} H(\bar{\varphi}(t))-\frac{H(\bar{\varphi}(t))}{q(t)} \int_{0}^{t} f^{\prime}(s) d s=0
\end{aligned}
$$

for $t>0$ and $\alpha(0)=0=\beta(0)$, we see $\alpha(t) \geq 0, \beta(t) \leq 0$ on $[0, \infty)$ and inequalities (10) are true.

3. Existence theorem. We define sequences $\left\{u_{n}\right\} \subset M,\left\{v_{n}\right\} \subset M$ by the recurrence formulas

$$
\begin{array}{ll}
u_{0}=\underline{\varphi}, & u_{n+1}=T\left(u_{n}\right), \\
v_{0}=\bar{\varphi}, & v_{n+1}=T\left(v_{n}\right)
\end{array}
$$

for $n=0,1,2, \ldots$

TheOrem 1. Let assumptions $\left(\mathrm{H}_{1}\right)-\left(\mathrm{H}_{6}\right)$ be fulfilled. Then the limits

$$
\lim _{n \rightarrow \infty} u_{n}(t)=: \underline{u}(t), \quad \lim _{n \rightarrow \infty} v_{n}(t)=: \bar{u}(t)
$$

exist for all $t \geq 0$. The functions $\underline{u}, \bar{u}$ are solutions of (2), and if $u$ is any solution of (2) then

$$
\underline{u}(t) \leq u(t) \leq \bar{u}(t) \quad \text { for } t \geq 0 .
$$


Proof. By Lemma 3 we have

$$
u_{0}(t) \leq u_{1}(t), \quad v_{1}(t) \leq v_{0}(t) \quad \text { for } t \geq 0 .
$$

Since $\alpha, \beta \in M$ and $\alpha(t) \leq \beta(t)$ for $t \geq 0$ implies $(T \alpha)(t) \leq(T \beta)(t)$ for $t \geq 0$, we deduce

$\underline{\varphi}(t)=u_{0}(t) \leq u_{1}(t) \leq \ldots \leq u_{n}(t) \leq \ldots \leq v_{n}(t) \leq \ldots \leq v_{1}(t) \leq v_{0}(t)=\bar{\varphi}(t)$

for $t \geq 0$ and $n \in \mathbb{N}$. Therefore the limits $\lim _{n \rightarrow \infty} u_{n}(t)=: \underline{u}(t), \lim _{n \rightarrow \infty} v_{n}(t)$ $=: \bar{u}(t)$ exist for all $t \geq 0, \varphi(t) \leq \underline{u}(t) \leq \bar{u}(t) \leq \bar{\varphi}(t)$ on $[0, \infty)$ and using the Lebesgue theorem we see that $\underline{u}, \bar{u}$ are solutions of (3) and $\underline{u}, \bar{u} \in M$.

If $u \in M$ is a solution of (3), by Lemma 2 we have

$$
\underline{\varphi}(t) \leq u(t) \leq \bar{\varphi}(t) \quad \text { for } t \geq 0
$$

and (11) follows by the monotonicity of $T$.

LEMMA 3. If (2) admits two different solutions $u$ and $v$, then $u(t) \neq v(t)$ for all $t>0$.

Proof. Let $u, v$ be two different solutions of (2). First, suppose there exists a $t_{1}>0$ such that $u(t)<v(t)$ for $t \in\left(0, t_{1}\right)$ and $u\left(t_{1}\right)=v\left(t_{1}\right)$. Since $H(u(t))-H(v(t))<0$ on $\left(0, t_{1}\right)$, we have

$K\left(u\left(t_{1}\right)\right)-K\left(v\left(t_{1}\right)\right)=\int_{0}^{t_{1}}\left(\frac{f(s)}{q(s)}-f^{\prime}(s) \int_{s}^{t_{1}} \frac{d z}{q(z)}\right)(H(u(s))-H(v(s))) d s<0$, contradicting $K\left(u\left(t_{1}\right)\right)=K\left(v\left(t_{1}\right)\right)$.

Secondly, suppose there exist $0<t_{1}<t_{2}$ such that $u\left(t_{n}\right)=v\left(t_{n}\right)(n=$ $1,2)$ and $u(t) \neq v(t)$ on $\left(t_{1}, t_{2}\right)$. Suppose

$$
u(t)<v(t) \quad \text { for } t \in\left(t_{1}, t_{2}\right) .
$$

Then $u^{\prime}\left(t_{1}\right)-v^{\prime}\left(t_{1}\right) \leq 0, u^{\prime}\left(t_{2}\right)-v^{\prime}\left(t_{2}\right) \geq 0, H(u(t))-H(v(t))<0$ on $\left(t_{1}, t_{2}\right)$, therefore

$$
\begin{aligned}
0 & \leq q\left(t_{2}\right) k\left(u\left(t_{2}\right)\right)\left(u^{\prime}\left(t_{2}\right)-v^{\prime}\left(t_{2}\right)\right)-q\left(t_{1}\right) k\left(u\left(t_{1}\right)\right)\left(u^{\prime}\left(t_{1}\right)-v^{\prime}\left(t_{1}\right)\right) \\
& =-\int_{t_{1}}^{t_{2}} f^{\prime}(s)(H(u(s))-H(v(s))) d s \leq 0
\end{aligned}
$$

and consequently, $f^{\prime}(t)=0$ on $\left[t_{1}, t_{2}\right]$. Hence $u^{\prime}\left(t_{1}\right)=v^{\prime}\left(t_{1}\right), f(t)=\mathrm{const}$ $(=: k)$ for $t \in\left[t_{1}, t_{2}\right]$ and

$$
K(u(t))-K(v(t))=\int_{t_{1}}^{t} \frac{k}{q(s)}(H(u(s))-H(v(s))) d s \quad \text { for } t \in\left[t_{1}, t_{2}\right] .
$$


Then we have

$$
0=K\left(u\left(t_{2}\right)\right)-K\left(v\left(t_{2}\right)\right)=\int_{t_{1}}^{t_{2}} \frac{k}{q(s)}(H(u(s))-H(v(s))) d s,
$$

which contradicts $H(u(t))-H(v(t)) \neq 0$ for $t \in\left(t_{1}, t_{2}\right)$.

\section{Bounded and unbounded solutions}

THEOREM 2. Let assumptions $\left(\mathrm{H}_{1}\right)-\left(\mathrm{H}_{6}\right)$ be fulfilled. Then

(i) some (and then any) solution of (2) is bounded if and only if

$$
\int_{0}^{\infty} \frac{d s}{q(s)}<\infty
$$

(ii) some (and then any) solution of (2) is unbounded if and only if

$$
\int_{0}^{\infty} \frac{d s}{q(s)}=\infty
$$

Pr o of. First observe that either $\int_{0}^{\infty} d s / q(s)<\infty$ or $\int_{0}^{\infty} d s / q(s)=\infty$.

Suppose $\int_{0}^{\infty} d s / q(s)<\infty$. Then according to Lemma 2 any solution of (3) (and by Lemma 1 also any solution of (2)) is bounded.

Suppose $\int_{0}^{\infty} d s / q(s)=\infty$ and let $u$ be a solution of $(2)$. Then

$$
K(u(t))=\int_{0}^{t}\left(\frac{f(s)}{q(s)}-f^{\prime}(s) \int_{s}^{t} \frac{d z}{q(z)}\right) H(u(s)) d s \quad \text { for } t \geq 0,
$$

and for $t \geq t_{1}$, where $t_{1}$ is a positive number, we have

$$
\begin{aligned}
K(u(t))= & \int_{0}^{t_{1}}\left(\frac{f(s)}{q(s)}-f^{\prime}(s) \int_{s}^{t} \frac{d z}{q(z)}\right) H(u(s)) d s \\
& +\int_{t_{1}}^{t}\left(\frac{f(s)}{q(s)}-f^{\prime}(s) \int_{s}^{t} \frac{d z}{q(z)}\right) H(u(s)) d s \\
\geq & H\left(u\left(t_{1}\right)\right) \int_{t_{1}}^{t}\left(\frac{f(s)}{q(s)}-f^{\prime}(s) \int_{s}^{t} \frac{d z}{q(z)}\right) d s \\
= & H\left(u\left(t_{1}\right)\right) f\left(t_{1}\right) \int_{t_{1}}^{t} \frac{d z}{q(z)} .
\end{aligned}
$$

Therefore $\lim _{t \rightarrow \infty} K(u(t))=\infty$ and $u$ is necessarily unbounded. 


\section{Uniqueness theorem}

TheOREm 3. Let assumptions $\left(\mathrm{H}_{1}\right)-\left(\mathrm{H}_{6}\right)$ be fulfilled. Assume that there exists $\varepsilon>0$ such that the modulus of continuity $\gamma(t)\left(:=\sup \left\{\left|q\left(t_{1}\right)-q\left(t_{2}\right)\right|\right.\right.$; $\left.\left.t_{1}, t_{2} \in[0, \varepsilon],\left|t_{1}-t_{2}\right| \leq t\right\}\right)$ of $q$ on $[0, \varepsilon]$ satisfies

$$
\limsup _{t \rightarrow 0^{+}} \gamma(t) / t<\infty \text {. }
$$

Then (2) admits a unique solution.

Pr o of. According to Lemma 1 and Theorem 1, it is sufficient to show that (3) admits a unique solution, that is, $\underline{u}=\bar{u}$, where $\underline{u}, \bar{u}$ are defined in Theorem 1. Since $0<\underline{u}(t) \leq \bar{u}(t)$ on $(0, \infty)$, we see that $\underline{u}^{\prime}(t)>0, \bar{u}^{\prime}(t)>0$ for $t>0$. Set $u_{1}=\underline{u}, u_{2}=\bar{u}, A_{i}=\lim _{t \rightarrow \infty} u_{i}(t)$ and $w_{i}=u_{i}^{-1}$, where $u_{i}^{-1}$ denotes the inverse function to $u_{i}(i=1,2)$. Then

$$
w_{i}^{\prime}(x)=q\left(w_{i}(x)\right) k(x)\left[\int_{0}^{x} f\left(w_{i}(s)\right) h(s) d s\right]^{-1} \quad \text { for } x \in\left(0, A_{i}\right), i=1,2
$$

and

$w_{i}(x)=\int_{0}^{x} q\left(w_{i}(s)\right) k(s)\left[\int_{0}^{s} f\left(w_{i}(z)\right) h(z) d z\right]^{-1} d s \quad$ for $x \in\left[0, A_{i}\right), i=1,2$.

Therefore, for $x \in\left[0, A_{1}\right)$ we have

(12) $(0 \leq) w_{1}(x)-w_{2}(x)$

$$
\begin{aligned}
= & \int_{0}^{x}\left(q\left(w_{1}(s)\right)-q\left(w_{2}(s)\right)\right) k(s)\left[\int_{0}^{s} f\left(w_{2}(z)\right) h(z) d z\right]^{-1} d s \\
& +\int_{0}^{x}\left\{q\left(w_{1}(s)\right) k(s)\left[\int_{0}^{s} f\left(w_{1}(z)\right) h(z) d z \int_{0}^{s} f\left(w_{2}(z)\right) h(z) d z\right]^{-1}\right. \\
& \left.\times \int_{0}^{s}\left(f\left(w_{2}(z)\right)-f\left(w_{1}(z)\right)\right) h(z) d z\right\} d s .
\end{aligned}
$$

Define $a=u_{1}(\varepsilon), X(x)=\max \left\{w_{1}(t)-w_{2}(t) ; 0 \leq t \leq x\right\}$ for $x \in[0, a]$. Suppose $X(x)>0$ on $(0, a]$. Then

$$
\left|q\left(w_{1}(x)\right)-q\left(w_{2}(x)\right)\right| \leq \gamma(X(x)) \quad \text { for } x \in[0, a]
$$

and using (12) we have

$$
w_{1}(x)-w_{2}(x) \leq(L X(x)+T \gamma(X(x))) V(x) \text { for } 0 \leq x \leq a,
$$

where

Hence

$$
T=\frac{1}{f(\varepsilon)}, \quad L=T^{2} \max _{t \in[0, \varepsilon]} f^{\prime}(t) \max _{t \in[0, \varepsilon]} q(t)
$$

$$
X(x) \leq(L X(x)+T \gamma(X(x))) V(x)
$$


and

$$
\frac{\gamma(X(x))}{X(x)} V(x) \geq(1-L V(x)) T^{-1} \quad \text { for } x \in(0, a] .
$$

By the assumption of Theorem 2, $\limsup _{x \rightarrow 0^{+}} \gamma(X(x)) / X(x)<\infty$, therefore $\lim _{x \rightarrow 0^{+}}(\gamma(X(x)) / X(x)) V(x)=0$, which contradicts the fact that $\lim _{x \rightarrow 0^{+}}(1-L V(x)) T^{-1}=T^{-1}$. This proves that there exists an interval $[0, b](0<b \leq \infty)$ such that $u_{1}=u_{2}$ on $[0, b]$.

Assume $u_{1} \not \equiv u_{2}$ on $[0, \infty)$ and let $[0, c]$ be the maximal interval where $u_{1}(t)=u_{2}(t)$. Define

$$
Y(t)=\max \left\{u_{2}(s)-u_{1}(s) ; c \leq s \leq t\right\} \quad \text { for } t \geq c .
$$

Then $Y(c)=0$ and $Y(t)>0$ for all $t>c$. Since

$K\left(u_{2}(t)\right)-K\left(u_{1}(t)\right)=\int_{c}^{t}\left(\frac{f(s)}{q(s)}-f^{\prime}(s) \int_{s}^{t} \frac{d z}{q(z)}\right)\left(H\left(u_{2}(s)\right)-H\left(u_{1}(s)\right)\right) d s$ for $t \geq c$, we have

$$
u_{2}(t)-u_{1}(t) \leq L_{1} Y(t) \int_{c}^{t}\left(\frac{f(s)}{q(s)}-f^{\prime}(s) \int_{s}^{t} \frac{d z}{q(z)}\right) d s \quad \text { for } t \in[c, c+1]
$$

where

$L_{1}=\max \left\{h(u) ; u \in\left[u_{1}(c), u_{2}(c+1)\right]\right\}\left[\min \left\{k(u) ; u \in\left[u_{1}(c), u_{2}(c+1)\right]\right\}\right]^{-1}$.

Hence

$$
Y(t)=L_{1} Y(t) \int_{c}^{t}\left(\frac{f(s)}{q(s)}-f^{\prime}(s) \int_{s}^{t} \frac{d z}{q(z)}\right) d s
$$

and

$$
1 \leq L_{1} \int_{c}^{t}\left(\frac{f(s)}{q(s)}-f^{\prime}(s) \int_{s}^{t} \frac{d z}{q(z)}\right) d s \quad \text { for } t \in(c, c+1],
$$

which is a contradiction. This completes the proof.

6. Dependence of solutions on the parameter. Consider the differential equation

$$
\left(q(t) k(u) u^{\prime}\right)^{\prime}=\lambda f(t) h(u) u^{\prime}, \quad \lambda>0,
$$

depending on the positive parameter $\lambda$. Assume that assumptions $\left(\mathrm{H}_{1}\right)^{-}$ $\left(\mathrm{H}_{6}\right)$ are satisfied. Set

$$
\underline{\varphi}(t ; \lambda)=V^{-1}\left(\lambda \int_{0}^{t} \frac{f(s)}{q(s)} d s\right), \quad \bar{\varphi}(t ; \lambda)=V^{-1}\left(\lambda f(0) \int_{0}^{t} \frac{d z}{q(z)}\right)
$$


and define

$$
\begin{array}{rlrl}
\left(T_{\lambda} u\right)(t) & =K^{-1}\left(\lambda \int_{0}^{t}\left(\frac{f(s)}{q(s)}-f^{\prime}(s) \int_{s}^{t} \frac{d z}{q(z)}\right) H(u(s)) d s\right), \\
u_{0}(t ; \lambda) & =\underline{\varphi}(t ; \lambda), & u_{n+1}(t ; \lambda)=\left(T_{\lambda} u_{n}\right)(t), \\
v_{0}(t ; \lambda) & =\bar{\varphi}(t ; \lambda), & v_{n+1}(t ; \lambda)=\left(T_{\lambda} v_{n}\right)(t)
\end{array}
$$

for $t \in[0, \infty), \lambda \in(0, \infty)$ and $n \in \mathbb{N}$.

TheOREm 4. Let assumptions $\left(\mathrm{H}_{1}\right)-\left(\mathrm{H}_{6}\right)$ be fulfilled. Then the limits

$$
\lim _{n \rightarrow \infty} u_{n}(t ; \lambda)=: \underline{u}(t ; \lambda), \quad \lim _{n \rightarrow \infty} v_{n}(t ; \lambda)=: \bar{u}(t ; \lambda)
$$

exist for $t \in[0, \infty)$ and $\lambda>0$. The functions $\underline{u}(t ; \lambda)$ and $\bar{u}(t ; \lambda)$ are solutions of $(13)$, and if $u(t ; \lambda)$ is any solution of $(13)$ then

$$
\underline{u}(t ; \lambda) \leq u(t ; \lambda) \leq \bar{u}(t ; \lambda) \quad \text { for } t \geq 0 .
$$

Moreover, for all $0<\lambda_{1}<\lambda_{2}$ we have

$$
\underline{u}\left(t ; \lambda_{1}\right)<\underline{u}\left(t ; \lambda_{2}\right), \quad \bar{u}\left(t ; \lambda_{1}\right)<\bar{u}\left(t ; \lambda_{2}\right) \quad \text { for } t>0 .
$$

Proof. The proof of the existence of the limits $\lim _{n \rightarrow \infty} u_{n}(t ; \lambda)$ and $\lim _{n \rightarrow \infty} v_{n}(t ; \lambda)$ and of $(15)$ is similar to the proof of Theorem 1 and therefore it is omitted here.

Let $0<\lambda_{1}<\lambda_{2}$. Then $\varphi\left(t ; \lambda_{1}\right)<\varphi\left(t ; \lambda_{2}\right), \bar{\varphi}\left(t ; \lambda_{1}\right)<\bar{\varphi}\left(t ; \lambda_{2}\right)$ and $\left(T_{\lambda_{1}} u\right)(t)<\left(T_{\lambda_{2}} u\right)(t)$ for each $u \in M$ and $t>0$. Since $H$ is strictly increasing on $[0, \infty)$, we have

$$
u_{n}\left(t ; \lambda_{1}\right)<u_{n}\left(t ; \lambda_{2}\right), \quad v_{n}\left(t ; \lambda_{1}\right)<v_{n}\left(t ; \lambda_{2}\right) \quad \text { for } t>0 \text { and } n \in \mathbb{N},
$$

and consequently,

$$
\underline{u}\left(t ; \lambda_{1}\right) \leq \underline{u}\left(t ; \lambda_{2}\right), \quad \bar{u}\left(t ; \lambda_{1}\right) \leq \bar{u}\left(t ; \lambda_{2}\right) \quad \text { for } t \geq 0 .
$$

If $v\left(t_{0} ; \lambda_{1}\right)=v\left(t_{0} ; \lambda_{2}\right)$ for a $t_{0}>0$, where $v$ is either $\underline{u}$ or $\bar{u}$, then in view of Lemma 1 we get

$$
\begin{aligned}
\lambda_{1} \int_{0}^{t_{0}}\left(\frac{f(s)}{q(s)}-f^{\prime}(s)\right. & \left.\int_{s}^{t_{0}} \frac{d z}{q(z)}\right) H\left(v\left(s ; \lambda_{1}\right)\right) d s \\
& =\lambda_{2} \int_{0}^{t_{0}}\left(\frac{f(s)}{q(s)}-f^{\prime}(s) \int_{s}^{t_{0}} \frac{d z}{q(z)}\right) H\left(v\left(s ; \lambda_{2}\right)\right) d s,
\end{aligned}
$$

contradicting $\lambda_{1}<\lambda_{2}$ and

$$
\left(\frac{f(t)}{q(t)}-f^{\prime}(t) \int_{t}^{t_{0}} \frac{d s}{q(s)}\right)\left(H\left(v\left(t ; \lambda_{1}\right)\right)-H\left(v\left(t ; \lambda_{2}\right)\right)\right) \leq 0 \quad \text { for } t \in\left(0, t_{0}\right] .
$$

Hence (16) is proved. 
THEOREM 5. Let the assumptions of Theorem 3 be fulfilled and $\int_{0}^{\infty} d s / q(s)$ $<\infty$. Then for each $a \in(0, \infty)$ there exists a unique $\lambda_{0}>0$ such that equation (13) with $\lambda=\lambda_{0}$ has a (necessarily unique) solution $u\left(t ; \lambda_{0}\right)$ with

$$
\lim _{t \rightarrow \infty} u\left(t ; \lambda_{0}\right)=a
$$

Proof. According to Theorem 3 equation (13) has for each $\lambda>0$ a unique solution $u(t ; \lambda)$, and by Theorem 1 this solution is bounded. Since $u(t ; \lambda)$ is strictly increasing in $t$ on $[0, \infty)$, we can define $g:(0, \infty) \rightarrow(0, \infty)$ by

$$
g(\lambda)=\lim _{t \rightarrow \infty} u(t ; \lambda) .
$$

According to Theorem $4, g$ is nondecreasing on $(0, \infty)$. If $g\left(\lambda_{1}\right)=g\left(\lambda_{2}\right)$ for some $0<\lambda_{1}<\lambda_{2}$, then

$$
\int_{0}^{\infty}\left(\frac{f(s)}{q(s)}-f^{\prime}(s) \int_{s}^{\infty} \frac{d z}{q(z)}\right)\left(H\left(u\left(s ; \lambda_{2}\right)\right)-H\left(u\left(s ; \lambda_{1}\right)\right)\right) d s=0,
$$

contradicting $H\left(u\left(t ; \lambda_{1}\right)\right)-H\left(u\left(t ; \lambda_{2}\right)\right)<0$ for $t \in(0, \infty)$. Hence $g$ is strictly increasing on $(0, \infty)$. To prove Theorem 5 it is enough to show that $g$ maps $(0, \infty)$ onto itself. First, we see from $\varphi(t ; \lambda) \leq u(t ; \lambda) \leq \bar{\varphi}(t ; \lambda)$ that $\lim _{\lambda \rightarrow 0^{+}} g(\lambda)=0$ and $\lim _{\lambda \rightarrow \infty} g(\lambda)=\infty$. Secondly, assume to the contrary

$$
\lim _{\lambda \rightarrow \lambda_{0}^{-}} g(\lambda)<\lim _{\lambda \rightarrow \lambda_{0}^{+}} g(\lambda)
$$

for a $\lambda_{0}>0$. Setting

$$
v_{1}(t)=\lim _{\lambda \rightarrow \lambda_{0}^{-}} u(t ; \lambda), \quad v_{2}(t)=\lim _{\lambda \rightarrow \lambda_{0}^{+}} u(t ; \lambda) \quad \text { for } t \geq 0,
$$

we get $v_{1} \neq v_{2}$. On the other hand, using the Lebesgue dominated convergence theorem as $\lambda \rightarrow \lambda_{0}^{-}$and $\lambda \rightarrow \lambda_{0}^{+}$in the equality

$$
u(t ; \lambda)=K^{-1}\left[\lambda \int_{0}^{t}\left(\frac{f(s)}{q(s)}-f^{\prime}(s) \int_{s}^{t} \frac{d z}{q(z)}\right) H(u(s ; \lambda)) d s\right]
$$

we see that

$$
v_{i}(t)=K^{-1}\left[\lambda_{0} \int_{0}^{t}\left(\frac{f(s)}{q(s)}-f^{\prime}(s) \int_{s}^{t} \frac{d z}{q(z)}\right) H\left(v_{i}(s)\right) d s\right]
$$

for $t \geq 0$ and $i=1,2$.

Therefore $v_{1}, v_{2}$ are solutions of (13) with $\lambda=\lambda_{0}$, contradicting the fact that equation (13) with $\lambda=\lambda_{0}$ has a unique solution. 


\section{References}

[1] F. V. Atkinson and L. A. Peletier, Similarity profiles of flows through porous media, Arch. Rational Mech. Anal. 42 (1971), 369-379.

[2] —, —, Similarity solutions of the nonlinear diffusion equation, ibid. 54 (1974), 373392.

[3] J. Bear, D. Zaslavsky and S. Irmay, Physical Principles of Water Percolation and Seepage, UNESCO, 1968.

[4] J. Goncerzewicz, H. Marcinkowska, W. Okrasiński and K. Tabisz, On the percolation of water from a cylindrical reservoir into the surrounding soil, Zastos. Mat. 16 (1978), 249-261.

[5] W. Okrasiński, Integral equations methods in the theory of the water percolation, in: Mathematical Methods in Fluid Mechanics, Proc. Conf. Oberwolfach 1981, Band 24, P. Lang, Frankfurt am Main 1982, 167-176.

[6] - On a nonlinear ordinary differential equation, Ann. Polon. Math. 49 (1989), $237-245$.

DEPARTMENT OF MATHEMATICAL ANALYSIS

FACULTY OF SCIENCE, PALACKÝ UNIVERSITY

Tř. SVOBODY 26

77146 OLOMOUC, CZECHOSLOVAKIA

Reçu par la Rédaction le 15.2.1991

Révisé le 30.6.1991 